\title{
Advances in tumor screening, imaging, and avatar technologies for high-grade serous ovarian cancer
}

\author{
Anders W. Ohman ${ }^{\dagger}$, Noor Hasan ${ }^{\dagger}$ and Daniela M. Dinulescu* \\ Division of Women's and Perinatal Pathology, Department of Pathology, Eugene Braunwald Research Center, Brigham and Women's Hospital, Harvard Medical \\ School, Boston, MA, USA
}

Edited by:

Elise Kohn, National Cancer Institute, USA

\section{Reviewed by:}

Tian-Li Wang, Johns Hopkins University School of Medicine, USA

Christina Annunziata, National Cancer Institute, USA

\section{*Correspondence:}

Daniela M. Dinulescu, Division of Women's and Perinatal Pathology, Department of Pathology, Eugene Braunwald Research Center, Brigham and Women's Hospital, Harvard

Medical School, 221 Longwood Avenue, 401A, Boston, MA 02215, USA

e-mail:ddinulescu@

rics.bwh.harvard.edu

${ }^{\dagger}$ Anders W. Ohman and Noor Hasan have contributed equally to this work.
The majority of high-grade serous ovarian carcinoma cases are detected in advanced stages when treatment options are limited. Surgery is less effective at eradicating the disease when it is widespread, resulting in high rates of disease relapse and chemoresistance. Current screening techniques are ineffective for early tumor detection and consequently, $B R C A$ mutations carriers, with an increased risk for developing high-grade serous ovarian cancer, elect to undergo risk-reducing surgery. While prophylactic surgery is associated with a significant reduction in the risk of cancer development, it also results in surgical menopause and significant adverse side effects. The development of efficient early-stage screening protocols and imaging technologies is critical to improving the outcome and quality of life for current patients and women at increased risk. In addition, more accurate animal models are necessary in order to provide relevant in vivo testing systems and advance our understanding of the disease origin and progression. Moreover, both genetically engineered and tumor xenograft animal models enable the preclinical testing of novel imaging techniques and molecularly targeted therapies as they become available. Recent advances in xenograft technologies have made possible the creation of avatar mice, personalized tumorgrafts, which can be used as therapy testing surrogates for individual patients prior to or during treatment. High-grade serous ovarian cancer may be an ideal candidate for use with avatar models based on key characteristics of the tumorgraft platform. This review explores multiple strategies, including novel imaging and screening technologies in both patients and animal models, aimed at detecting cancer in the early-stages and improving the disease prognosis.

Keywords: fallopian tubal secretory epithelial cell, high-grade serous carcinoma, genetically engineered mouse models, avatar models, serous tubal intraepithelial carcinoma, near-infrared fluorescence, the Cancer Genome Atlas, BRCA

\section{TREATMENT APPROACHES FOR PATIENTS AND HIGH-RISK WOMEN}

Ovarian cancer is the most lethal gynecological malignancy, with the majority of high-grade serous carcinoma (HGSC) cases being diagnosed in late stages of disease [for a comprehensive review see Bast (1)]. The current American Cancer Society statistics estimates that the 5 -year survival is $44 \%$ when all disease stages are included but declines to $25 \%$ if only advanced stage cases are considered (1-3). Initial treatment for HGSC patients involves debulking surgery, which typically includes a combination of hysterectomy, bilateral salpingo-oophorectomy, and removal of the omentum, followed by therapy using platinum compounds and taxanes $(4,5)$. While platinum-based chemotherapy improves patient survival, the treatment is also very toxic; in addition, disease relapse following treatment and the acquisition of platinum chemoresistance are frequent events, suggesting a need for alternative treatment

Abbreviations: FR- $\alpha$, folate receptor $\alpha$; FTSEC, fallopian tubal secretory epithelial cell; GEMM, genetically engineered mouse models; HGSC, high-grade serous carcinoma; NIRF, near-infrared fluorescence; STIC, serous tubal intraepithelial carcinoma; TCGA, the Cancer Genome Atlas. modalities (6). One strategy involves the addition of bevacizumab, a monoclonal antibody targeting VEGF-A, which has been tested together with standard chemotherapy in the setting of platinumresistant recurrent disease. Indeed, the combination of an antiangiogenic compound and single-agent chemotherapy improved objective response rates and progression free survival in comparison with chemotherapy alone, but the overall survival trend was not significant (7). An alternative strategy, which is aimed at reducing both the treatment toxicity and recurrence rates, proposes the administration of lower doses of carboplatin plus paclitaxel given once a week for 18 weeks instead of standard doses administered every 3 weeks for six cycles. Interestingly, this modified weekly regimen of reduced chemotherapeutic doses has recently been found to not only be an effective option for first-line treatment but, most importantly, to be associated with an enhanced quality of life as assessed physically, socially, emotionally, and functionally (8).

New treatment approaches could be beneficial not only for patients but also for women at increased risk for developing the disease. A recent study, which included a broadly spanning, exome-wide analysis of both ovarian cancer somatic and germline mutations, has estimated that more than $20 \%$ of women 
likely have an inherited predisposition to ovarian cancer (9). The most studied mutations involve the BRCA family of tumor suppressor genes, which confer an increased risk for developing breast and ovarian cancers (10). The lifetime risk for developing breast cancer in BRCA mutation carriers varies between 56 and $84 \%$ while the ovarian cancer risk ranges from 36$46 \%$ to $10-27 \%$ for women with BRCA1 and BRCA2 mutations, respectively (11). In addition, lifetime risks for both breast and ovarian cancers due to BRCA mutations appear to have increased over time, possibly due to lower physical activity and higher BMIs (12). When assessing individual patient risks for ovarian cancer it is important to note that irrespective of genetic risk, women with irregular menstrual cycles experience a 2.4 times greater incidence of death due to ovarian cancer (13).

\section{QUALITY OF LIFE AFTER PROPHYLACTIC SURGERY IN HIGH-RISK WOMEN}

The mean age at the time of diagnosis for the average population is 63 years; by comparison, the mean age for BRCA mutation carriers is considerably lower at 50.8 years. For both groups, the cancer risk increases with age, especially after menopause. Consequently, the current recommendation for $B R C A$ carriers is to undergo risk-reducing surgery once childbearing is completed, since they tend to be diagnosed at an earlier age than sporadic ovarian cancer (14). Standard prophylactic surgical options include bilateral mastectomy and salpingo-oophorectomy, which confer the most substantial reduction in cancer risk and increase in life expectancy $(10,15,16)$. Studies looking at the efficacy of bilateral prophylactic salpingo-oophorectomy in BRCA mutation carriers concluded that it not only resulted in a $96 \%$ reduction in the risk of developing coelomic epithelial cancers but also decreased the breast cancer risk by $53 \%$ in comparison with $B R C A$ mutation carriers who chose not to undergo the procedure (14). Despite the drastic risk-reduction associated with prophylactic surgery, it is far from an ideal treatment approach. In addition to being stripped of their inherently female characteristics, women who choose to undergo a combination of mastectomy, bilateral salpingo-oophorectomy, and hysterectomy are faced with a significant decrease in their quality of life, premature menopause, hot flashes, decreased cognition and sexual function, and increased risk of osteoporosis and cardiac mortality $(14,17-19)$. The use of hormone therapy and other medications may mitigate some of these adverse effects. Nevertheless, hormone replacement therapy is controversial, especially in high-risk women, as it has been linked to an increased risk of breast cancer (20). While an elective oophorectomy procedure may correlate negatively with life expectancy in women at average risk, the procedure remains beneficial for women at increased risk (21). Despite the possible negative physiological and psychological impacts of prophylactic oophorectomy, women at increased risk for developing HGSC report less anxiety about developing the disease, which they believe compensates for undesirable side effects (16). The primary cause of depression reported amongst post-surgical women is due to sexual dysfunction (21). In addition, BRCA mutation carriers may also experience the fear of transmitting a hereditary disease to their children. Women with these reproductive concerns could choose to investigate alternative methods, including in vitro fertilization and screening of embryos via preimplantation genetic diagnosis in order to eliminate the chance of transmitting faulty BRCA genes to their children (17).

\section{NON-SURGICAL RISK-REDUCTION APPROACHES IN BRCA MUTATION CARRIERS}

There are limited risk-reducing approaches for women with $B R C A$ mutations who choose not to undergo prophylactic surgery (22). Current strategies include early breast cancer screening consisting of annual mammogram and breast MRI. In addition, gynecologic cancer screening consists of baseline transvaginal ultrasound and CA-125 serum level measurements followed by frequent monitoring using ROCA evaluation protocols. Breast cancer screening results in early tumor detection and a survival advantage; in contrast, ovarian cancer screening has yet to be associated with a significant reduction in mortality (23). For example, $63 \%$ of ovarian cancers detected were stage IIC or higher in a comprehensive study of over 6000 high-risk women (23). This can be partially attributed to the fact that current screening options are limited and not best suited to detect early-stage cancers (24). An alternative strategy involves the use of chemopreventive methods, including selective estrogen receptor inhibitors, tamoxifen and raloxifene, in addition to oral contraceptives (15). Oral contraceptives have indeed been shown to decrease the risk of developing ovarian cancer in the general population (25). However, the use of oral contraceptives in BRCA mutation carriers, while beneficial for ovarian cancer prevention, may be associated with an increased risk for developing breast cancers $(26,27)$.

\section{DEVELOPMENT OF IMAGING TECHNOLOGIES AIMED AT EARLY DETECTION AND IMPROVING PATIENT OUTCOME}

As mentioned above, the vast majority of both familial and spontaneous HGSC cases are diagnosed in advanced stages, at which point the prognosis is poor (28). Because there are few clear early symptoms, developing effective methods for early detection is paramount to improving long-term patient survival (29). Current methods of detection include non-invasive screenings using serum CA-125, ultrasound, sonography, CT, and MRI scans (30, 31). A laparoscopic procedure may also be used to provide an image of the lower abdominal organs, as well as attain a biopsy, which is necessary in order to confirm cancer diagnosis. These tools can give information regarding the size, composition, and location of the tumor and whether it has spread, which will be important for disease staging and treatment plans $(28,32)$. In terms of early detection, it is important to note that current imaging technologies are not able to distinguish precursor lesions inside the fallopian tube or ovary, two of the tumor initiation sites for HGSC. Novel in vivo imaging devices, such as confocal microlaparoscopes, which are instrumental in providing live images of abnormal regions and guiding biopsies, may be better equipped to assist with early tumor diagnosis (33). Images obtained by these probes in real-time during surgery have shown a clear distinction between normal and abnormal regions within the ovarian surface epithelium (33). Furthermore, studies using a flexible microlaparoscope have demonstrated the ability to provide highresolution images of early stage cancer inside the fallopian tube in the intra-operative setting, thereby facilitating both an earlier 
diagnosis and accurate disease staging (34). Since these procedures have shown merit in surgical settings, they may become a viable complementary option to traditional biopsies as future modalities of disease confirmation (33). Nevertheless, the specificity and sensitivity of this method have yet to be determined in clinical trials and there are also drawbacks associated with this procedure. For example, the image quality produced by the microlaparoscope is diminished when compared to traditional laparoscopes due to a smaller scope size, focal distance, and a reduced light output. Procedural complications may also arise from a lack of tight correlation between the movement of the instrument handle and instrument movement in the surgical field due to its increased flexibility (35). Other considerations include a reduced imaging depth, which may limit the ability to view cells below the tissue surface layer, as well as the development of non-toxic and non-mutagenic contrast-enhancing agents (33). Nevertheless, the device can successfully image organs in vivo without major complications (33) and needs to be carefully evaluated in future clinical trials.

Another widely used primary diagnostic tool for women seeking evaluation of a pelvic mass is transvaginal sonography (TVS). Traditional TVS technologies are being replaced by contrast enhanced transvaginal sonography (CE-TVS) and transvaginal color Doppler sonography (TV-CDS), which can better depict the tumor morphology by analyzing its microvasculature (31). In a study aimed at evaluating the diagnostic ability of contrast enhanced 3-dimensional power Doppler sonography (CE-3D) relative to conventional 3D Doppler sonography, the CE-3D technology showed $95.6 \%$ accuracy in distinguishing between benign and cancerous tumors compared to $86.7 \%$ accuracy for the conventional method (36). Contrast-enhancing agents coupled with transvaginal and Doppler sonography may merit further investigation as an early screening tool for women at risk. It is worth mentioning that the development of selective means for tumor imaging is critical not only for early detection but also for improving patient outcome (29). Thus, the use of selective intraoperative tumor imaging devices has the potential to both improve disease staging and enhance precision during cytoreductive surgery as it allows for better visualization of tumors (37). For example, a combination of functional in vivo and anatomical ex vivo X-ray micro-computed tomography $(\mu \mathrm{CT})$ can provide a highly detailed three-dimensional analysis of the tumor micromorphology, vascularization, and accurately quantify relative blood volumes (rBV) in tumors, which can further inform treatment plans (38). Most importantly, the study has found a direct correlation between microvascular parameters (i.e., vessel size, the complexity of vessel branching) and tumor angiogenesis and aggressiveness (38).

Further advances have been made in ultrasound technology as well. Photoacoustic imaging is an emerging technology based on the photoacoustic effect, which is generated when tissues are pulsed with non-ionizing lasers. This results in a transient thermoelastic expansion and emission of an acoustic wave, which is detected by ultrasonic transducers and converted into images (39). A study evaluating biodegradable photoacoustic imaging agents in animal models of ovarian cancer found that cellulose nanoparticles produced high contrast signals. Interestingly, cellulose nanoparticles demonstrated a significant increase in signal when compared to gold nanoparticles, which are commonly used in photoacoustic imaging. Unfortunately, this imaging agent only proved to be biodegradable ex vivo. For the purpose of reducing toxicity and to facilitate clinical translation, further research will need to focus on nanoparticles that biodegrade within the mammalian circulatory system (40).

A strong emphasis is currently placed on evaluating imaging agents that are safe to use in patients and allow the visualization of early or recurrent tumors. Current research in ovarian cancer investigates the use of the folate receptor $\alpha$ (FR- $\alpha$ ) and HER-2 as targeted agents for tumor-specific fluorescence imaging. Thus, FR- $\alpha$ is overexpressed in the majority of epithelial ovarian cancers, especially in HGSC tumors with a high risk of recurrence. Increased FR- $\alpha$ expression is detected not only in primary tumors but also in metastatic foci and recurrent tumors (41). Most importantly, chemotherapy does not appear to significantly alter FR- $\alpha$ expression in patient tumors $(37,41)$. In addition, FR- $\alpha$-targeted fluorescent agents are able to selectively enhance imaging of tumor cells (37). PPF, an FR-targeted probe that is well suited for both PET and optical imaging was investigated in vivo in a trial using primary cell xenografts, in vitro with primary human ovarian cancer cells, and ex vivo with omentum removed from xenografts (42). PPF injected either intraperitoneally or intravenously was able to identify FR-positive primary HGSC tumors and their metastases to the omentum. As FR is overexpressed in HGSC, FR-targeted probes, such as PPF, may bear great utility in the clinical setting. This method could be ideal for guiding surgery due to the noninvasive, high-resolution, real-time images it produces (42). The unique features of this novel imaging tool may prove useful for ovarian cancer detection and monitoring. Furthermore, fluorescence imaging using FR- $\alpha$-targeted agents could play a critical role during debulking surgery, as current methods of imaging are not tumor-specific.

Fluorescence imaging has been shown to detect a greater number of tumors when compared to conventional methods. Interestingly, a vinblastine folate-targeted drug, vintafolide, when used in combination with a diagnostic imaging tool, etarfolatide, may merit attention as a means to advance personalized treatments. Thus, etarfolatide imaging has been used successfully to select patients with FR-positive platinum-resistant ovarian tumors who may benefit from folate-targeted therapy (43). This novel combination of folate-targeted agents for imaging and treatment resulted in a marked increase in progression free survival for platinumresistant patients. Based on the highly selective nature of this treatment, the drug efficacy was reported to be greater and its toxicity decreased compared to standard therapy (43). An alternative strategy for selective tumor imaging involves the use of the HER-2 biomarker, which is overexpressed in advanced HGSC cases (44). Imaging using HER-2-targeted magnetic iron oxide nanoparticles allows both magnetic resonance and optical imaging of peritoneal tumors when used in orthotopic ovarian xenograft models (45). This technology, which enables tumor imaging with high specificity and resolution, can be instrumental in drug delivery and image-guided surgery $(44,45)$. A third strategy involves the development of near-infrared fluorescence (NIRF) imaging technologies, which have been successfully tested in vivo in both pancreatic and ovarian cancer models as an alternative to ultrasound, CT, and MRI scans (46). Gene expression profiling was instrumental in 
identifying proteases relevant to tumors, which further enabled the development of protease-specific NIRF probes. Such probes can provide not only a higher resolution for molecular-guided detection of early tumors but also the ability to distinguish between inflammation and cancer (46). Similarly, an alpha(v)beta(3-) integrin targeted NIRF probe was used successfully in ovarian xenograft models to optimize debulking surgery (47). Furthermore, the increased target to background ratio, high sensitivity (95\%), specificity (88\%), and diagnostic accuracy (96.5\%) of this imaging system suggest that the NIRF-targeted platform is well suited for clinical translation and may be able to provide highly accurate images of small tumor lesions that are otherwise difficult to detect (47).

Plasma tumor biomarkers for the detection of early tumors and precursor lesions have been difficult to identify. CA-125 is presently used to help diagnose ovarian cancer, mainly for late stage cases, and to predict the chance of tumor recurrence (48). Nevertheless, CA-125 is not always a reliable biomarker for early stage tumors due to its lack of sensitivity (48). Large screening studies are currently underway to determine the sensitivity and specificity of a combined monitoring protocol using serum CA125 levels and TVS for early diagnosis. The screening is based on an improved algorithm designed by Dr. Steven Skates to identify cancer risk based on rising trends in individual CA-125 levels (1). An alternative strategy involves the combined evaluation of multiple biomarkers in addition to CA-125. Research suggests that the addition of HE4, leptin, prolactin, osteopontin, insulin-like growth factor-II, CEA, and soluble vCAM cancer biomarkers to CA-125 serum surveillance protocols may result in a better diagnostic reliability when compared to CA-125 alone $(1,25)$. It is worth noting that recent studies suggest that a significant proportion of HGSC tumors originate from precursor lesions [serous tubal intraepithelial carcinoma (STIC)] located within the fallopian tube rather than the ovary, and this is the case especially in BRCA women $(1,49)$. Consequently, the development of serum screening tests and methods of diagnostic imaging need to include markers characteristic for the fallopian tube/fimbria in addition to the ovary. Currently, small early tumors within the fallopian tube cannot be detected via ultrasound or by measuring serum CA-125 levels (50). Comprehensive screening of such lesions through endometrial cytological testing may be a promising method for the early detection of HGSC in high-risk women and BRCA mutation carriers. Otsuka et al. reported that tumor cells shed from tubal STICs could be detected through careful examination of endometrial cytological samples (50). This would enhance the early detection rates for HGSC tumors as the occurrence of false positives through cytological testing appears to be low (50). Interestingly, this pilot study was able to detect malignant cells in five patients for whom imaging results were normal; three of them presented with no symptoms and were later diagnosed with early-stage HGSCs (50). In addition to further confirming the tubal site of origin for HGSC cases, the study also reported a 4-fold increase in the number of high-grade serous tumors being detected using this method when compared to other ovarian cancer subtypes (50). In contrast, the direct testing of cervicovaginal cytological samples yielded positive results in only one of five patients, suggesting that this is not an efficient means of detecting early-stage HGSC tumors.

\section{GENERATION OF IMPROVED ANIMAL MODELS THAT CLOSELY RESEMBLE HGSC}

In addition to aiding in the development of new imaging techniques, murine models of ovarian cancer are integral to drug development and disease pathogenesis studies $(46,51)$. While several genetically engineered mouse models (GEMM) have been previously developed for endometrioid ovarian cancer (52-54), clinically relevant models for HGSC have been difficult to generate. This could be due to model designs based solely on a traditional view of disease pathogenesis, such as the ovarian origin hypothesis. New clinical protocols, which involve the sequential sectioning and examination of the fimbrial (SEE-FIM) end of the fallopian tube pioneered by Dr. Christopher Crum, have identified precursor lesions for HGSC arising in secretory cells of the fallopian tube, namely p53 signatures and STICs (55-58). In addition, several groups have been instrumental in leading efforts for refining murine HGSC models. Recently, the first genetic model of de novo HGSC originating in fallopian tubal secretory epithelial cells (FTSEC) has been generated, which recapitulates key genetic alterations (BRCA, TP53, and PTEN) and precursor lesions (STICs) that are hallmarks of the human disease (59). In addition to offering mechanistic insight into the origin and pathogenesis of HGSC, this model provides a platform to explore tumor sensitivity to novel therapeutic agents and diagnostic imaging methods that include the distal fallopian tube in addition to the ovary (59). Recently, a second genetically engineered model of HGSC was described using the Ovgp-1 promoter to target SV40 large $\mathrm{T}$-antigen-induced tumorigenesis in the fallopian tube (60). This model also displays neoplastic lesions of the fallopian tube that resemble human STICs and p53 signatures. The murine ovarian carcinomas have molecular characteristics that strongly resemble the human disease as well. Furthermore, gene expression analysis studies of Ovgp-1-driven tumors have identified a novel biomarker, topoisomerase II-alpha, which is overexpressed with mutant TP53 in both murine and human STICs and HGSCs but not in normal adjacent tissues (60). Most importantly, this model provides independent support for the hypothesis that HGSC may be primarily tubal in origin and mirrors the clinical progression of human HGSCs (61). The development of FTSEC-driven animal models will be instrumental in providing a platform to test newly emerging data from The Cancer Genome Atlas (TCGA) in an in vivo relevant system. The goal of the research community is to develop ovarian cancer models that recapitulate not only novel genetic/genomic alterations but also the histopathology and clinical behavior of HGSCs. Resolving the pathogenesis of HGSC and its precursor lesions will likely enable more efficient methods for early detection, tumor imaging, and cancer prevention. Furthermore, by using a combination of murine model studies and epidemiological data from patients, it will be important to determine if premenopausal women with $B R C A$ mutations can be offered risk-reduction surgery in a multi-step procedure without undergoing surgical menopause and loss of fertility in their younger years.

As a complement to genetically engineered models, personalized patient-derived murine xenografts ("avatar mice") have been developed, which are able to more accurately predict tumor responses to therapy. Xenograft tumor models have been used 
for decades to examine the behavior of various types of therapies within a living system (62). They can closely resemble the molecular and histological characteristics of the human cancer they are derived from $(63,64)$ and demonstrate clinical relevance by predicting the activity and effects of trial therapies (65). However, their predictive ability varies dramatically based on the cancer being studied, and multiple therapies, which tested well in xenografts, did not ultimately result in successful clinical trials (66-68). While traditional xenografts are created by generating and then engrafting established cell lines (51), a subcategory of xenografts produced by direct transfer of patient tumor tissue into immunocompromised mice (also known as explant xenografts or tumorgrafts) has mimicked the drug effects seen in humans much more closely than cell line xenografts (69). Furthermore, one study of personalized tumorgrafts involving a broad range of humanderived tumors and anticancer therapies demonstrated a positive clinical predictive value for drug resistance over $90 \%$ of the time (70). Unlike human cell cultures, which tend to result in increasingly homogenous populations with successive passages, direct tissue transplants more accurately represent the heterogeneous makeup and genetic diversity of the original tumor, including its relative cell proportions and overall genomic profile $(71,72)$. These tumor sections can be implanted orthotopically within the homologous source tissue in addition to being dispersed into the body cavity via intraperitoneal injection, as is the case for cell line xenografts $(73,74)$. Using these techniques, avatar models have been generated that closely recapitulate the tumor of a specific donor patient. This has led to the identification of personalized therapeutic regimens by creating a tailored stand-in for patient tumors prior to or alongside treatment (75). The use of tumorgraft testing surrogates, which are generated by using a specific patient's own tissue, has increased over the last decade. This trend was initiated by a recent study that described the use of xenograft technologies to create personalized tumorgrafts for a total of 14 patients with a variety of cancer types (76). The study results identified optimal, non-obvious treatment choices with a high rate of clinical success (76). This process has been performed with similar success in models of lung (77), pancreatic (78), prostate (79), breast $(80,81)$, and fallopian tube (82) cancers. In addition to being highly representative of the morphology and progression of human cancers, it was found that the success of tumor engraftment is by itself a prognostic indicator of disease outcome for women with newly diagnosed breast cancer (80).

While GEMM and xenografts both strive to generate accurate models of human disease and their usage at times overlaps, they have individual features best suited to distinct roles in cancer research. Tumors that develop from a xenograft retain the natural genetic alterations derived from the original source $(63,83)$. Conversely, GEM models must recreate these changes based on the result of investigation or hypothesis, and therefore they often cannot replicate the complexity and genomic diversity found in patient tumors (74). In cancers with high variance in molecular alterations between patients, tumorgrafts should be used to test therapies in models that more accurately represent individual tumors (77), since generalized results from GEMM studies will not be applicable. Personalized tumorgrafts have also been used to identify changes in drug resistance at specific stages of disease by grafting repeatedly from the same patient at different time points (63). Furthermore, avatar models could also allow the preemptive identification of new treatment strategies necessary when a patient develops resistance to clinically available therapies (84). Being able to determine tumor sensitivity and drug resistance for each individual patient upfront would allow oncologists to attempt experimental treatments with a higher probability of success while retaining conventional therapies as an option (85). In contrast, GEMMs have attributes superior to avatar models when it comes to studying the origin of the disease, precursor lesions, tumor progression, and the contribution of the immune system to cancer pathogenesis by allowing the inducible targeting of key genes in a tissue-specific manner in immunocompetent mice (86). There are several challenges in creating avatar mice relative to GEM and cell line xenograft models, including the need for surgical extraction of adequate tumor samples from the patient, ideally including accessory tissue (72), and a high rate of implantation failure (85). While tumor heterogeneity is represented, the microenvironment is typically not. This drawback, combined with the use of immunocompromised mice, restricts how similarly avatar models behave when compared to human disease. Such limitations could be overcome by incorporating recent xenograft advancements. The tumor microenvironment can be retained in a xenograft by grafting stroma alongside the tumor (75) and the use of "humanized" models preserve immune system functionality after engraftment (87). Besides technical concerns, there is a high financial barrier for creating new avatar lines (75). However, once tissues have been extracted and implanted, human tumors can be serially passaged in mice, archived, and later repropagated from tumor banks (82). The generation of tumor banks enables the repeated testing and study of patient tumors from a small number of original extractions.

Further development of representative mouse models of HGSC is an urgent need for the field (88) and tumorgrafts have attributes suited to this subtype. HGSCs are characterized by rapid metastasis (89) and tumorgraft models were reported to metastasize to regions similar to those seen in patients (90). HGSCs are also characterized by genomic instability (51) and tumorgrafts were shown to accurately retain the genomic profile of the original patient sample in animal models throughout multiple grafts (72). Avatar mice have been generated for fallopian tube carcinoma (82), which supports their viability for ovarian cancer, particularly in conjunction with the tubal origin hypothesis (91). This year, the first large-scale tumorgraft mouse study of ovarian cancer was published, consisting of 168 engrafted models from patient samples, which were representative of the entire spectrum of the disease (83). As in previous tumorgraft studies, the models closely resembled the patients they were derived from. The majority of models that developed ascites originated from patients with ascites, which is notable as the development of ascites is characteristic of HGSC (89). Consistent with prior comparisons between cell line xenografts and donor patient platinum sensitivity in ovarian cancer (92), all of the ovarian models tested for platinum sensitivity had the same type of response as the donor patient (83). It has been suggested that the tumor microenvironment may play a role in the high rate of relapse and increased drug resistance seen in HGSC (93). These ovarian tumorgrafts strongly 
resemble the source microenvironment by inducing the formation of tumor stroma (83). As shown in a larger tumorgraft study (72), the ovarian models closely resembled the source patient's genomic alterations after engraftment and the grafts implanted in clinically relevant sites. Finally, just as demonstrated previously (80), the initial success of a graft tended to correlate negatively with patient survival (83). This extensive study demonstrates the feasibility of ovarian tumorgrafts as patient surrogates, particularly given the reasonably accurate representation of the disease diversity (94). Consequently, the generation of avatar mouse models for HGSC is expected to assist oncologists with establishing individual resistance profiles quickly in a surrogate model following biopsy and informing patients of therapy choices in real-time (85).

Avatar tumorgrafts have been found to be highly predictive models clinically and the generation of such models can aid with drug design on an individual patient basis $(67,76)$. The use of avatar models alongside patients in concurrent clinical trials has already been proposed (75), though it is noted that variance in how accurately these strains reflect human disease, including the contribution of the immune system, can be a confounding factor. In recent years, there has been renewed interest in harnessing the immune system to target cancers $(95,96)$, often achieved by blocking the inhibitors of immune reactions elicited by tumor cells. This approach has led to considerable success, such as the use of ipilimumab immunotherapy in metastatic melanoma (96). Ipilimumab is a monoclonal antibody against CTLA-4 and promotes effective antitumor targeting by cytotoxic $\mathrm{T}$ lymphocytes. Therapies targeting CTLA-4 have also been investigated in ovarian cancer; a combined treatment, which involves blockade of CTLA4 and PD- 1 and boosts the immune system, was found to induce tumor rejection in $75 \%$ of tumor models examined (97). In addition, the endothelium of many tumors may function as a primary defense against immune system activation by creating a physical protective barrier for the tumor and resisting immune cell invasion (98). It remains to be seen whether the development of effective immunotherapies will increase the efficacy of conventional therapies and achieve durable remissions in patients (96). Notably, it was recently demonstrated that traditional therapeutic regimens could be modified to effectively recruit the immune system in the setting of platinum-resistant relapsed disease (6). While platinum and paclitaxel are often delivered at maximum tolerable doses, the dose-dense chemotherapy study demonstrates that delivery of conventional drugs in low doses within frequent intervals can enhance natural antitumor immune responses and reduce immunosuppression, thus leading to increased treatment efficacy (6). Thus, a dose-dense chemotherapy regimen was successful in promoting the antitumor $\mathrm{CD}^{+}{ }^{+} \mathrm{T}$-cell response in both mouse models and patients and reduced the tumor ability to suppress the immune system. This bodes well for further optimization of such treatments for individual needs, particularly as an extended weekly, dose-dense carboplatin and paclitaxel regimen has been shown to be effective in heavily pre-treated, recurrent, platinum-resistant ovarian cancer patients (99). Clearly, optimizing these treatments for patients requires a clinically translatable graft model. Xenograft models with an implanted functional human immune system have been previously developed to investigate viral and immune disease (100). Interestingly, such models were shown to be functional in cancer as well (87). A key benefit of GEMMs over xenografts has always been that they retain normal immune function (51). However, a xenograft with a humanized immune system, which is interacting with human tumor cells, may be more translatable to designing immunotherapies for patients based on individual needs. While GEMMs may ultimately prove more useful than avatar models for understanding the intricate details of HGSC origin and progression, personalized tumorgrafts will be key for the design of individual therapeutic regimens.

\section{CONCLUSIONS}

Advances in tumor screening and imaging may help determine the optimal time to employ risk-reducing surgical approaches in women at high risk for HGSC, including BRCA mutation carriers. Prophylactic surgery offers the most significant reduction in the risk of developing breast and ovarian cancers, as current surveillance methods are not effective enough to lend support for ovarian conservation in premenopausal women at high risk. Bilateral salpingo-oophorectomy is, however, associated with multiple physiological and psychosocial side effects that may contribute to a decrease in the quality of life and a loss of fertility in younger women. Consequently, the use of endometrial cytological testing in high-risk women or improved in vivo imaging devices (i.e., confocal microlaparoscopes, photoacoustic imaging, $\mu \mathrm{CT}$, and contrast enhanced 3D Doppler sonography) could prove to be more effective for both early detection and treatment. In addition, alternative strategies for tumor-specific imaging, which involve the use of FR- $\alpha$-targeted fluorescent agents, HER-2-targeted magnetic iron oxide nanoparticles, or protease-specific NIRF probes, merit further investigation as selective tools for early tumor detection, monitoring, and image-guided surgery. It is worth noting that animal models are a valuable tool in ovarian cancer research for the purpose of developing and testing novel imaging technologies, biomarkers, and experimental treatments. However, there are currently a limited number of animal models available for HGSC. Advances in tumor xenograft technologies have enabled the development of personalized avatar mouse models, which have emerged as an ideal drug-testing platform, especially in concurrent clinical trials. Additionally, tumorgrafts appear to have qualities well suited to model HGSC. We suggest that avatars have the potential to improve patient outcome and quality of life by reducing the cost and toxicity of ineffective imaging and treatment.

\section{AUTHOR CONTRIBUTIONS}

Anders W. Ohman, Noor Hasan, and Daniela M. Dinulescu wrote the manuscript.

\section{ACKNOWLEDGMENTS}

This work is supported by grants awarded to Daniela M. Dinulescu by the DOD OCRP (W81XWH-10-1-0263, W81XWH14-1-0092, and W81XWH-14-1-0205), American Cancer Society (RSG-13-083-01-TBG), NIH (1R03CA189462-01), Ovarian Cancer Research Fund Liz Tilberis award, Burroughs-Wellcome Fund Career Award in the Biomedical Sciences 1005320.01, V Foundation for Cancer Research Scholar Award, Rivkin Foundation, and the Mildred Moorman Ovarian Cancer Research Fund. 


\section{REFERENCES}

1. Bast RC. Molecular approaches to personalizing management of ovarian cancer. Ann Oncol (2011) 8:viii5-15. doi:10.1093/annonc/mdr516

2. Siegel R, Ma J, Zou Z, Jemal A. Cancer statistics, 2014. CA Cancer J Clin (2014) 64(1):9-29. doi:10.3322/caac.21208

3. Baldwin LA, Huang B, Miller RW, Tucker T, Goodrich ST, Podzielinski I, et al. Ten-year relative survival for epithelial ovarian cancer. Obstet Gynecol (2012) 120:612-8. doi:10.1097/AOG.0b013e318264f794

4. Cherry C, Ropka M, Lyle J, Napolitano L, Daly MB. Understanding the needs of women considering risk-reducing salpingo-oophorectomy. Cancer Nurs (2013) 36:E33-8. doi:10.1097/NCC.0b013e3182642cb5

5. American Cancer Society. Surgery for Ovarian Cancer. (2013) [cited 2014 Aug 11]. Available from: http://www.cancer.org/cancer/ovariancancer/ detailedguide/ovarian-cancer-treating-surgery

6. Chang C, Hsu M, Wu C, Lai Y, Wang C, Yang C, et al. Dose-dense chemotherapy improves mechanisms of antitumor immune response. Cancer Res (2013) 73(1):119-27. doi:10.1158/0008-5472.CAN-12-2225

7. Pujade-Lauraine E, Hilpert F, Weber B, Reuss A, Poveda A, Kristensen G, et al. Bevacizumab combined with chemotherapy for platinum-resistant recurrent ovarian cancer: the AURELIA open-label randomized phase III trial. J Clin Oncol (2014) 32(13):1302-8. doi:10.1200/JCO.2013.51.4489

8. Pignata S, Scambia G, Katsaros D, Gallo C, Pujade-Lauraine E, De Placido S, et al. Carboplatin plus paclitaxel once a week versus every 3 weeks in patients with advanced ovarian cancer (MITO-7): a randomised, multicentre, openlabel, phase 3 trial. Lancet Oncol (2014) 15(4):396-405. doi:10.1016/S14702045(14)70049-X

9. Kanchi KL, Johnson KJ, Lu C, McLellan MD, Leiserson MD, Wendl MC, et al. Integrated analysis of germline and somatic variants in ovarian cancer. Nat Commun (2014) 5:3156. doi:10.1038/ncomms4156

10. van Roosmalen MS, Verhoef LC, Stalmeier PF, Hoogerbrugge N, van Daal WA. Decision analysis of prophylactic surgery or screening for BRCA1 mutation carriers: a more prominent role for oophorectomy. J Clin Oncol (2002) 20:2092-100. doi:10.1200/JCO.2002.08.035

11. Rebbeck TR, Kauff ND, Domchek SM. Meta-analysis of risk reduction estimates associated with risk-reducing salpingo-oophorectomy in BRCA1 or BRCA2 mutation carriers. J Natl Cancer Inst (2009) 101:80-7. doi:10.1093/ jnci/djn442

12. King MC, Marks JH, Mandell JB, New York Breast Cancer Study Group. Breast and ovarian cancer risks due to inherited mutations in BRCA1 and BRCA2. Science (2003) 302:643-6. doi:10.1126/science.1088759

13. Cohn B. Irregular Menses Linked to High Risk of Ovarian Cancer. San Diego, CA: American Association for Cancer Research (2014).

14. Rebbeck TR, Lynch HT, Neuhausen SL, Narod SA, Van't Veer L, Garber JE, et al. Prophylactic oophorectomy in carriers of BRCA1 or BRCA2 mutations. N Engl J Med (2002) 346:1616-22. doi:10.1056/NEJMoa012158

15. Salhab M, Bismohun S, Mokbel K. Risk-reducing strategies for women carrying BRCA1/2 mutations with a focus on prophylactic surgery. BMC Womens Health (2010) 10:28. doi:10.1186/1472-6874-10-28

16. Tiller K, Meiser B, Butow P, Clifton M, Thewes B, Friedlander M, et al. Psychological impact of prophylactic oophorectomy in women at increased risk of developing ovarian cancer: a prospective study. Gynecol Oncol (2002) 86:212-9. doi:10.1006/gyno.2002.6737

17. Stan DL, Shuster LT, Wick MJ, Swanson CL, Pruthi S, Bakkum-Gamez JN. Challenging and complex decisions in the management of the BRCA mutation carrier. J Womens Health (Larchmt) (2013) 22:825-34. doi:10.1089/jwh.2013. 4407

18. Erekson EA, Martin DK, Ratner ES. Oophorectomy: the debate between ovarian conservation and elective oophorectomy. Menopause (2013) 20:110-4. doi:10.1097/gme.0b013e31825a27ab

19. Kauff ND, Satagopan JM, Robson ME, Scheuer L, Hensley M, Hudis CA, et al. Risk-reducing salpingo-oophorectomy in women with BRCA1 or BRCA2 mutation. N Engl J Med (2002) 346:1609-15. doi:10.1056/NEJMoa020119

20. Hou N, Hong S, Wang W, Olopade OI, Dignam JJ, Huo D. Hormone replacement therapy and breast cancer: heterogeneous risks by race, weight, and breast density. J Natl Cancer Inst (2013) 105:1365-72. doi:10.1093/jnci/djt207

21. Robson M, Hensley M, Barakat R, Brown C, Chi D, Poynor E, et al. Quality of life in women at risk for ovarian cancer who have undergone risk-reducing oophorectomy. Gynecol Oncol (2003) 89:281-7. doi:10.1016/S0090-8258(03) 00072-6
22. Kauff ND, Barakat RR. Risk-reducing salpingo-oophorectomy in patients with germline mutations in BRCA1 or BRCA2. J Clin Oncol (2007) 25:2921-7. doi:10.1200/JCO.2007.11.3449

23. Hogg R, Friedlander M. Biology of epithelial ovarian cancer: implications for screening women at high genetic risk. J Clin Oncol (2004) 22:1315-27. doi:10.1200/JCO.2004.07.179

24. Kehoe SM, Kauff ND. Screening and prevention of hereditary gynecologic cancers. Semin Oncol (2007) 34:406-10. doi:10.1053/j.seminoncol.2007.07.004

25. Gadducci A, Sergiampietri C, Tana R. Alternatives to risk-reducing surgery for ovarian cancer. Ann Oncol (2013) 24(Suppl 8):viii47-53. doi:10.1093/annonc/ mdt311

26. Modan B, Hartge P, Hirsh-Yechezkel G, Chetrit A, Lubin F, Beller U, et al. Parity, oral contraceptives, and the risk of ovarian cancer among carriers and noncarriers of a BRCA1 or BRCA2 mutation. N Engl J Med (2001) 345:235-40. doi:10.1056/NEJM200107263450401

27. Narod SA, Dube MP, Klijn J, Lubinski J, Lynch HT, Ghadirian P, et al. Oral contraceptives and the risk of breast cancer in BRCA1 and BRCA2 mutation carriers. J Natl Cancer Inst (2002) 94:1773-9. doi:10.1093/jnci/94.23.1773

28. Release MAN. Promising Screening Tool for Early Detection of Ovarian Cancer. Houston, TX: MD Anderson (2013).

29. Jacobs IJ, Menon U. Progress and challenges in screening for early detection of ovarian cancer. Mol Cell Proteomics (2004) 3:355-66. doi:10.1074/mcp. R400006-MCP200

30. Iyer VR, Lee SI. MRI, CT, and PET/CT for ovarian cancer detection and adnexal lesion characterization. AJR Am J Roentgenol (2010) 194:311-21. doi:10.2214/AJR.09.3522

31. Fleischer AC, Lyshchik A, Hirari M, Moore RD, Abramson RG, Fishman DA. Early detection of ovarian cancer with conventional and contrast-enhanced transvaginal sonography: recent advances and potential improvements. J Oncol (2012) 2012:302858. doi:10.1155/2012/302858

32. American Cancer Society. How is Ovarian Cancer Diagnosed? (2014) [cited 2014 Aug 11]. Available from: http://www.cancer.org/cancer/ovariancancer/ detailedguide/ovarian-cancer-diagnosis

33. Tanbakuchi AA, Udovich JA, Rouse AR, Hatch KD, Gmitro AF. In vivo imaging of ovarian tissue using a novel confocal microlaparoscope. Am J Obstet Gynecol (2010) 202(1):.e1-9. doi:10.1016/j.ajog.2009.07.027

34. Wu T-Y, Schafer R, Rouse AR, Gmitro AF. Confocal microlaparoscope for imaging the fallopian tube. Proc. SPIE 8217, Endoscopic Microscopy VII. Bellingham, WA: SPIE (2012). 82170 O p. doi:10.1117/12.906430

35. Demco L. Complications of microlaparoscopy and awake laparoscopy. JSLS (2003) 7:141-5.

36. Kupesic S, Kurjak A. Contrast-enhanced, three-dimensional power Doppler sonography for differentiation of adnexal masses. Obstet Gynecol (2000) 96:452-8. doi:10.1016/S0029-7844(00)00923-6

37. van Dam GM, Themelis G, Crane LM, Harlaar NJ, Pleijhuis RG, Kelder W, et al. Intraoperative tumor-specific fluorescence imaging in ovarian cancer by folate receptor-alpha targeting: first in-human results. Nat Med (2011) 17:1315-9. doi: $10.1038 / \mathrm{nm} .2472$

38. Ehling J, Theek B, Gremse F, Baetke S, Mockel D, Maynard J, et al. Micro-CT imaging of tumor angiogenesis: quantitative measures describing micromorphology and vascularization. Am J Pathol (2014) 184:431-41. doi:10.1016/j. ajpath.2013.10.014

39. Aguirre J, Morales-Dalmau J, Funk L, Jara F, Turon P, Durduran T. The potential of photoacoustic microscropy as a tool to characterize the in vivo degradation of surgical structures. Biomed Opt Express (2014) 5(8):2856-69. doi:10.1364/BOE.5.002856

40. Jokerst JV, Van de Sompel D, Bohndiek SE, Gambhir SS. Cellulose nanoparticles are a biodegradable photoacoustic contrast agent for use in living mice. Photoacoustics (2014) 2(3):119-27. doi:10.1016/j.pacs.2014.07.001

41. Kalli KR, Oberg AL, Keeney GL, Christianson TJ, Low PS, Knutson KL, et al. Folate receptor alpha as a tumor target in epithelial ovarian cancer. Gynecol Oncol (2008) 108:619-26. doi:10.1016/j.ygyno.2007.11.020

42. Liu T, Steward J, MacDonald T, Chen J, Clarke B, Shi J, et al. Biologically - targeted detection of primary and micro-metastatic ovarian cancer. Theranostics (2013) 3(6):420-7. doi:10.7150/thno.6413

43. Serpe L, Gallicchio M, Canaparo R, Dosio F. Targeted treatment of folate receptor-positive platinum-resistant ovarian cancer and companion diagnostics, with specific focus on vintafolide and etarfolatide. Pharmgenomics Pers Med (2014) 7:31-42. doi:10.2147/PGPM.S58374 
44. Hsieh CY, Chen CA, Chou CH, Lai KP, Jeng YM, Kuo ML, et al. Overexpression of Her-2/NEU in epithelial ovarian carcinoma induces vascular endothelial growth factor C by activating NF-kappa B: implications for malignant ascites formation and tumor lymphangiogenesis. J Biomed Sci (2004) 11:249-59. doi:10.1007/BF02256568

45. Satpathy M, Wang L, Zielinski R, Qian W, Lipowska M, Capala J, et al. Active targeting using HER-2-affibody-conjugated nanoparticles enabled sensitive and specific imaging of orthotopic HER-2 positive ovarian tumors. Small (2014) 10(3):544-55. doi:10.1002/smll.201301593

46. von Burstin J, Eser S, Seidler B, Meining A, Bajbouj M, Mages J, et al. Highly sensitive detection of early-stage pancreatic cancer by multimodal nearinfrared molecular imaging in living mice. Int J Cancer (2008) 123:2138-47. doi:10.1002/ijc.23780

47. Harlaar NJ, Kelder W, Sarantopoulos A, Bart J, Themelis G, van Dam GM, et al. Real-time near infrared fluorescence (NIRF) intra-operative imaging in ovarian cancer using an alpha(v)beta(3-)integrin targeted agent. Gynecol Oncol (2013) 128:590-5. doi:10.1016/j.ygyno.2012.12.011

48. Karabudak AA, Hafner J, Shetty V, Chen S, Secord AA, Morse MA, et al. Autoantibody biomarkers identified by proteomics methods distinguish ovarian cancer from non-ovarian cancer with various CA-125 levels. J Cancer Res Clin Oncol (2013) 139:1757-70. doi:10.1007/s00432-013-1501-6

49. Erickson BK, Conner MG, Landen CN Jr. The role of the fallopian tube in the origin of ovarian cancer. Am J Obstet Gynecol (2013) 209(5):409-14. doi:10.1016/j.ajog.2013.04.019

50. Otsuka I, Kameda S, Hoshi K. Early detection of ovarian and fallopian tube cancer by examination of cytological samples from the endometrial cavity. $\mathrm{Br}$ J Cancer (2013) 109:603-9. doi:10.1038/bjc.2013.402

51. Ricci F, Broggini M, Damia G. Revisiting ovarian cancer preclinical models: implications for a better management of the disease. Cancer Treat Rev (2013) 39:561-8. doi:10.1016/j.ctrv.2013.01.005

52. Dinulescu DM, Ince TA, Quade BJ, Shafer SA, Crowley D, Jacks T. Role of $\mathrm{K}$-ras and Pten in the development of mouse models of endometriosis and endometrioid ovarian cancer. Nat Med (2005) 11:63-70. doi:10.1038/nm1173

53. Wu R, Hendrix-Lucas N, Kuick R, Zhai Y, Schwartz DR, Akyol A, et al. Mouse model of human ovarian endometrioid adenocarcinoma based on somatic defects in the Wnt/beta-catenin and PI3K/Pten signaling pathways. Cancer Cell (2007) 11:321-33. doi:10.1016/j.ccr.2007.02.016

54. Wu R, Baker SJ, Hu TC, Norman KM, Fearon ER, Cho KR. Type I to type II ovarian carcinoma progression: mutant Trp53 or Pik3ca confers a more aggressive tumor phenotype in a mouse model of ovarian cancer. Am J Pathol (2013) 182:1391-9. doi:10.1016/j.ajpath.2012.12.031

55. Kindelberger DW, Lee Y, Miron A, Hirsch MS, Feltmate C, Medeiros F, et al. Intraepithelial carcinoma of the fimbria and pelvic serous carcinoma: evidence for a causal relationship. Am J Surg Pathol (2007) 31:161-9. doi:10.1097/01. pas.0000213335.40358.47

56. Lee Y, Miron A, Drapkin R, Nucci MR, Medeiros F, Saleemuddin A, et al. A candidate precursor to serous carcinoma that originates in the distal fallopian tube. J Pathol (2007) 211:26-35. doi:10.1002/path.2091

57. Medeiros F, Muto MG, Lee Y, Elvin JA, Callahan MJ, Feltmate C, et al. The tubal fimbria is a preferred site for early adenocarcinoma in women with familial ovarian cancer syndrome. Am J Surg Pathol (2006) 30:230-6. doi:10.1097/01.pas.0000180854.28831.77

58. Przybycin CG, Kurman RJ, Ronnett BM, Shih Ie M, Vang R. Are all pelvic (nonuterine) serous carcinomas of tubal origin? Am J Surg Pathol (2010) 34:1407-16. doi:10.1097/PAS.0b013e3181ef7b16

59. Perets R, Wyant GA, Muto KW, Bijron JG, Poole BB, Chin KT, et al. Transformation of the fallopian tube secretory epithelium leads to high-grade serous ovarian cancer in Brca;Tp53;Pten models. Cancer Cell (2013) 24(6):751-65. doi:10.1016/j.ccr.2013.10.013

60. Sherman-Baust CA, Kuhn E, Valle BL, Shih IM, Kurman RJ, Wang TL, et al. A genetically engineered ovarian cancer mouse model based on fallopian tube transformation mimics human high-grade serous carcinoma development. $J$ Pathol (2014) 233(3):228-37. doi:10.1002/path.4353

61. Kurman RJ, Shih I. The origin and pathogenesis of epithelial ovarian cancer - a proposed unifying theory. Am J Surg Pathol (2010) 34(3):433-43. doi:10.1097/PAS.0b013e3181cf3d79

62. Kelland LR. Of mice and men: values and liabilities of the athymic nude mouse model in anticancer drug development. Eur J Cancer (2004) 40:827-36. doi:10.1016/j.ejca.2003.11.028
63. Massazza G, Lucchini V, Tomasoni A, Peccatori F, Lampasona V, Giudici G, et al. Malignant behavior and resistance to cisplatin of human ovarian carcinoma xenografts established from the same patient at different stages of the disease. Cancer Res (1991) 51:6358-62.

64. Massazza G, Tomasoni A, Lucchini V, Allavena P, Erba E, Colombo N, et al. Intraperitoneal and subcutaneous xenografts of human ovarian carcinoma in nude mice and their potential in experimental therapy. Int J Cancer (1989) 44:494-500. doi:10.1002/ijc.2910440320

65. Hu L, Hofmann J, Holash J, Yancopoulos GD, Sood AK, Jaffe RB. Vascular endothelial growth factor trap combined with paclitaxel strikingly inhibits tumor and ascites, prolonging survival in a human ovarian cancer model. Clin Cancer Res (2005) 11:6966-71. doi:10.1158/1078-0432.CCR-05-0910

66. Voskoglou-Nomikos T, Pater JL, Seymour L. Clinical predictive value of the in vitro cell line, human xenograft, and mouse allograft preclinical cancer models. Clin Cancer Res (2003) 9:4227-39.

67. Garber K. From human to mouse and back: 'tumorgraft' models surge in popularity. J Natl Cancer Inst (2009) 101:6-8. doi:10.1093/jnci/djn481

68. Kerbel RS. Human tumor xenografts as predictive preclinical models for anticancer drug activity in humans: better than commonly perceived-but they can be improved. Cancer Biol Ther (2003) 2:S134-9. doi:10.4161/cbt.213

69. Tentler JJ, Tan AC, Weekes CD, Jimeno A, Leong S, Pitts TM, et al. Patientderived tumour xenografts as models for oncology drug development. Nat Rev Clin Oncol (2012) 9:338-50. doi:10.1038/nrclinonc.2012.61

70. Fiebig HH, Maier A, Burger AM. Clonogenic assay with established human tumour xenografts: correlation of in vitro to in vivo activity as a basis for anticancer drug discovery. Eur J Cancer (2004) 40:802-20. doi:10.1016/j.ejca.2004. 01.009

71. Moro M, Bertolini G, Tortoreto M, Pastorino U, Sozzi G, Roz L. Patient-derived xenografts of non small cell lung cancer: resurgence of an old model for investigation of modern concepts of tailored therapy and cancer stem cells. J Biomed Biotechnol (2012) 2012:568567. doi:10.1155/2012/568567

72. Monsma DJ, Monks NR, Cherba DM, Dylewski D, Eugster E, Jahn H, et al. Genomic characterization of explant tumorgraft models derived from fresh patient tumor tissue. J Transl Med (2012) 10:125. doi:10.1186/1479-5876-10125

73. Zhang X, Claerhout S, Prat A, Dobrolecki LE, Petrovic I, Lai Q, et al. A renewable tissue resource of phenotypically stable, biologically and ethnically diverse, patient-derived human breast cancer xenograft models. Cancer Res (2013) 73:4885-97. doi:10.1158/0008-5472.CAN-12-4081

74. Richmond A, Su Y. Mouse xenograft models vs GEM models for human cancer therapeutics. Dis Model Mech (2008) 1:78-82. doi:10.1242/dmm.000976

75. Malaney P, Nicosia SV, Dave V. One mouse, one patient paradigm: new avatars of personalized cancer therapy. Cancer Lett (2014) 344(1):1-12. doi:10.1016/j.canlet.2013.10.010

76. Hidalgo M, Bruckheimer E, Rajeshkumar NV, Garrido-Laguna I, De Oliveira E, Rubio-Viqueira B, et al. A pilot clinical study of treatment guided by personalized tumorgrafts in patients with advanced cancer. Mol Cancer Ther (2011) 10:1311-6. doi:10.1158/1535-7163.MCT-11-0233

77. Dong X, Guan J, English JC, Flint J, Yee J, Evans K, et al. Patient-derived first generation xenografts of non-small cell lung cancers: promising tools for predicting drug responses for personalized chemotherapy. Clin Cancer Res (2010) 16:1442-51. doi:10.1158/1078-0432.CCR-09-2878

78. Villarroel MC, Rajeshkumar NV, Garrido-Laguna I, De Jesus-Acosta A, Jones S, Maitra A, et al. Personalizing cancer treatment in the age of global genomic analyses: PALB2 gene mutations and the response to DNA damaging agents in pancreatic cancer. Mol Cancer Ther (2011) 10:3-8. doi:10.1158/1535-7163. MCT-10-0893

79. Zhao H, Thong A, Nolley R, Reese SW, Santos J, Ingels A, et al. Patientderived tissue slice grafts accurately depict response of high-risk primary prostate cancer to androgen deprivation therapy. J Transl Med (2013) 11:199. doi:10.1186/1479-5876-11-199

80. DeRose YS, Wang G, Lin YC, Bernard PS, Buys SS, Ebbert MT, et al. Tumor grafts derived from women with breast cancer authentically reflect tumor pathology, growth, metastasis and disease outcomes. Nat Med (2011) 17:1514-20. doi: $10.1038 / \mathrm{nm} .2454$

81. Litzenburger BC, Creighton CJ, Tsimelzon A, Chan BT, Hilsenbeck SG, Wang T, et al. High IGF-IR activity in triple-negative breast cancer cell lines and tumorgrafts correlates with sensitivity to anti-IGF-IR therapy. Clin Cancer Res (2011) 17:2314-27. doi:10.1158/1078-0432.CCR-10-1903 
82. Khabele D, Fadare O, Liu AY, Wilson AJ, Wass E, Osteen K, et al. An orthotopic model of platinum-sensitive high grade serous fallopian tube carcinoma. Int $\mathrm{J}$ Clin Exp Pathol (2012) 5:37-45.

83. Weroha SJ, Becker MA, Enderica-Gonzalez S, Harrington SC, Oberg AL, Maurer MJ, et al. Tumorgrafts as in vivo surrogates for women with ovarian cancer. Clin Cancer Res (2014) 20(5):1288-97. doi:10.1158/1078-0432.CCR-13-2611

84. Dennis C. Mouse 'avatars' could aid pancreatic cancer therapy. Nature (2012) doi:10.1038/nature.2012.10259

85. Morelli MP, Calvo E, Ordonez E, Wick MJ, Viqueira BR, Lopez-Casas PP, et al. Prioritizing phase I treatment options through preclinical testing on personalized tumorgraft. J Clin Oncol (2012) 30:e45-8. doi:10.1200/JCO.2011.36. 9678

86. Lengyel E, Burdette JE, Kenny HA, Matei D, Pilrose J, Haluska P, et al. Epithelial ovarian cancer experimental models. Oncogene (2014) 33(28):3619-33. doi:10.1038/onc.2013.321

87. Vatakis DN, Bristol GC, Kim SG, Levin B, Liu W, Radu CG, et al. Using the BLT humanized mouse as a stem cell based gene therapy tumor model. J Vis Exp (2012) (70):e4181. doi:10.3791/4181

88. Mullany LK, Richards JS. Minireview: animal models and mechanisms of ovarian cancer development. Endocrinology (2012) 153:1585-92. doi:10.1210/en. 2011-2121

89. Lengyel E. Ovarian cancer development and metastasis. Am J Pathol (2010) 177:1053-64. doi:10.2353/ajpath.2010.100105

90. Thong AE, Zhao H, Ingels A, Valta MP, Nolley R, Santos J, et al. Tissue slice grafts of human renal cell carcinoma: an authentic preclinical model with high engraftment rate and metastatic potential. Urol Oncol (2014) 32(1):.e23-30. doi:10.1016/j.urolonc.2013.05.008

91. Crum CP, Drapkin R, Miron A, Ince TA, Muto M, Kindelberger DW, et al. The distal fallopian tube: a new model for pelvic serous carcinogenesis. Curr Opin Obstet Gynecol (2007) 19:3-9. doi:10.1097/GCO.0b013e328011a21f

92. Harrap KR, Jones M, Siracky J, Pollard LA, Kelland LR. The establishment, characterization and calibration of human ovarian carcinoma xenografts for the evaluation of novel platinum anticancer drugs. Ann Oncol (1990) 1:65-76.

93. Chien J, Kuang R, Landen C, Shridhar V. Platinum-sensitive recurrence in ovarian cancer: the role of tumor microenvironment. Front Oncol (2013) 3:251. doi:10.3389/fonc.2013.00251

94. Tothill RW, Tinker AV, George J, Brown R, Fox SB, Lade S, et al. Novel molecular subtypes of serous and endometrioid ovarian cancer linked to clinical outcome. Clin Cancer Res (2008) 14(16):5198-208. doi:10.1158/1078-0432. CCR-08-0196

95. Sharma P, Wagner K, Wolchok JD, Allison JP. Novel cancer immunotherapy agents with survival benefit: recent successes and next steps. Nat Rev Cancer (2011) 1(11):805-12. doi:10.1038/nrc3153

96. Mellman I, Coukos G, Dranoff G. Cancer immunotherapy comes of age. Nature (2011) 480(7378):480-9. doi:10.1038/nature10673

97. Duraiswamy J, Kaluza KM, Freeman GJ, Coukos G. Dual blockade of PD-1 and CTLA-4 combined with tumor vaccine effectively restores T-cell rejection function in tumors. Cancer Res (2013) 73(12):3591-603. doi:10.1158/00085472.CAN-12-4100

98. Motz GT, Santoro SP, Wang LP, Garrabrant T, Lastra RR, Hagemann IS, et al. Tumor endothelium FasL establishes a selective immune barrier promoting tolerance in tumors. Nat Med (2014) 20:607-15. doi:10.1038/nm.3541

99. Sharma R, Graham J, Mitchell H, Brooks A, Blagden S, Gabra H. Extended weekly dose-dense paclitaxel/carboplatin is feasible and active in heavily pre-treated platinum-resistant recurrent ovarian cancer. Br J Cancer (2009) 100(5):707-12. doi:10.1038/sj.bjc.6604914

100. Shultz LD, Brehm MA, Garcia-Martinez JV, Greiner DL. Humanized mice for immune system investigation: progress, promise and challenges. Nat Rev Immunol (2012) 12(11):786-98. doi:10.1038/nri3311

Conflict of Interest Statement: The authors declare that the research was conducted in the absence of any commercial or financial relationships that could be construed as a potential conflict of interest.

Received: 05 June 2014; accepted: 27 October 2014; published online: 18 November 2014.

Citation: Ohman AW, Hasan N and Dinulescu DM (2014) Advances in tumor screening, imaging, and avatar technologies for high-grade serous ovarian cancer. Front. Oncol. 4:322. doi: 10.3389/fonc.2014.00322

This article was submitted to Women's Cancer, a section of the journal Frontiers in Oncology.

Copyright (c) 2014 Ohman, Hasan and Dinulescu. This is an open-access article distributed under the terms of the Creative Commons Attribution License (CC BY). The use, distribution or reproduction in other forums is permitted, provided the original author(s) or licensor are credited and that the original publication in this journal is cited, in accordance with accepted academic practice. No use, distribution or reproduction is permitted which does not comply with these terms. 